\title{
Lyapunov Characteristic Exponent Maps for Multi-Body Space Systems Analysis
}

\author{
Daniele Pagnozzi * and James D. Biggs ${ }^{\dagger}$ \\ Advanced Space Concepts Laboratory, Mechanical and Aerospace Engineering Department, \\ University of Strathclyde, Glasgow, UK
}

\begin{abstract}
This paper investigates the natural dynamics of a space multibody system in orbit around a celestial body using modern dynamical systems theory. In particular Lyapunov Characteristic Exponent (LCE) maps, which are used in celestial mechanics and fluid dynamics, are here applied to a multi-body system to analyse different qualitative behaviours. Complemented with phase diagrams and Poincare maps, LCE maps are shown to be an extremely useful global visualisation tool. Such a map reduces the order of the problem, condensing quantities of information into a lower-dimensional image. Here, a simple example is considered to demonstrate the usefulness of LCE maps with the aim of using it on more complex, realistic cases in the future. For this simple example a Hamiltonian formulation is derived to facilitate an analytical analysis of the systems equilibria and their nonlinear stability and to aid the validation of the numerical results.
\end{abstract}

Keywords: Multibody, Rigid Body Dynamics, Space, Lyapunov Characteristic Exponent, Hamiltonian

\section{INTRODUCTION}

SPACE multibody systems refer to a large class of complex spacecraft which include space robotic and Nmanipulator systems. As a practical example, consider space robotic systems such as the free-flying or free-floating robots described in ${ }^{1}$ and. $^{2}$ The capability to perform a number of construction, inspection and repair tasks is fundamental. In order to realise the potential of such space multi-body systems, issues on controllability, maneuver planning and tracking design must be addressed. However, such issues are particularly hard to tackle as the dynamics are highly non-linear and the attitude and orbital dynamics are coupled, see $,{ }^{3},{ }^{45}$ and $^{6}$ for recent overviews.

The literature on space (or free) multi-body systems can be loosely split into two categories; those which address the analysis of their natural dynamics and those which address the design of motion planning and control methodologies. The latter covers the majority of the publications and do not directly exploit the global dynamics of the system in their design. Referring to an analysis of free multibody dynamics from a mathematical or geometric approach, Sreenath, et. al. ${ }^{78}$ provide a study of the dynamics of free coupled planar rigid bodies based on the manipulation and analysis of the Hamiltonian structure in the reduced phase space and examine equilibria and their stability using the energy-Casimir method. It is notable that they illustrate that around homoclinic orbits, chaotic motion arises when the system is perturbed by the presence of a third body. Moreover, they find the existence of two periodic orbits near the stable equilibrium. Grossman et al. ${ }^{9}$ provide a three dimensional study of a free two-body system.

Extensions to space multi-body systems have been addressed in Bogoyavlenskil, ${ }^{10}$ who presented all the possible particular cases in which a multibody system subject to a gravity field is an integrable problem. Other approaches model space multi-body systems as chains of point masses rather than rigid bodies. Guerman, ${ }^{11}$ addressed the problem of tethered satellites, studying a model consisting of point masses connected by light rods and spherical hinges, assuming the orbital dynamics as uncoupled with the attitude dynamics. Moreover, the system centers of mass are assumed to be moving along circular orbits in order to find all sets of equilibria of a generic N-link chain. In, ${ }^{12},{ }^{13}$ and, ${ }^{14}$ similar systems were studied under the action of a

*PhD Student, daniele.pagnozzi@strath.ac.uk, AIAA Student.

${ }^{\dagger}$ Lecturer, james.biggs@strath.ac.uk 
central gravity field. However, they consider the coupling between orbital and attitude dynamics obtaining relative equilibria and studying their stability.

Research on space multi-body systems reveals a very complex dynamics and often assumptions and simplifications are made in order to study the system. In particular, only the free planar two body problem is an integrable one in the general case. When additional bodies or external forces are introduced, the problem is in general non-integrable and as shown in, ${ }^{8}$ chaotic behaviors may arise.

A deep understanding of the underlying dynamics of a system can significantly enhance the system design with respect to the architecture, motion planning and control. This paper is motivated by the need for an efficient global visualisation tool that is designed specifically for the analysis of space multibody systems and can be used to inform system design.

Modern computers facilitate our understanding of complex dynamics by using numerical methods. Therefore, in this paper Lyapunov Characteristic Exponent (LCE) Maps are used to study the dynamics of multiple rigid-body systems in a central gravitational field. LCEs are a modern analysis numerical tool which has been used in several other scientific fields, typically and with successful results in fluid dynamics ${ }^{15,16}$ and celestial mechanics, ${ }^{17-19}$ to identify regular and chaotic motion regions. However, to the best of the authors' knowledge, the application of LCEs to space robotic systems is new. LCE maps are shown to be an extremely useful global visualisation tool. Such a map reduces the order of the problem, condensing quantities of information into a lower-dimensional image. LCE maps have two main advantages; first they can be generated directly by iterative integration of the dynamics, with no need to manipulate the equations or perform a change of coordinates. Second, they are a visual tool and they provide an almost-global description of the dynamics. Hence, LCE maps are a promising tool which could be systematically used in engineering design process. A more detailed description will follow in the paper.

In order to investigate the benefits of LCEs when applied to space multi-body systems, a very simple example is considered and the results are compared with the ones obtained with standard analysis methods. The intent is to move on to more complex, realistic cases in the future.

The contribution of the paper is as follows:

- Derivation of the Hamiltonian dynamics of a planar two-body system in a central gravitational field;

- Identification of equilibria and non-linear stability analysis using Dirichelt's theorem for Hamiltonian systems;

- Representation of the system's dynamics by Phase Portaits and Poincaré sections to highlight different qualitative behaviours of the system;

- Generation of the LCE map and discussion on its relation to previous results.

\section{THE PHYSICAL MODEL}

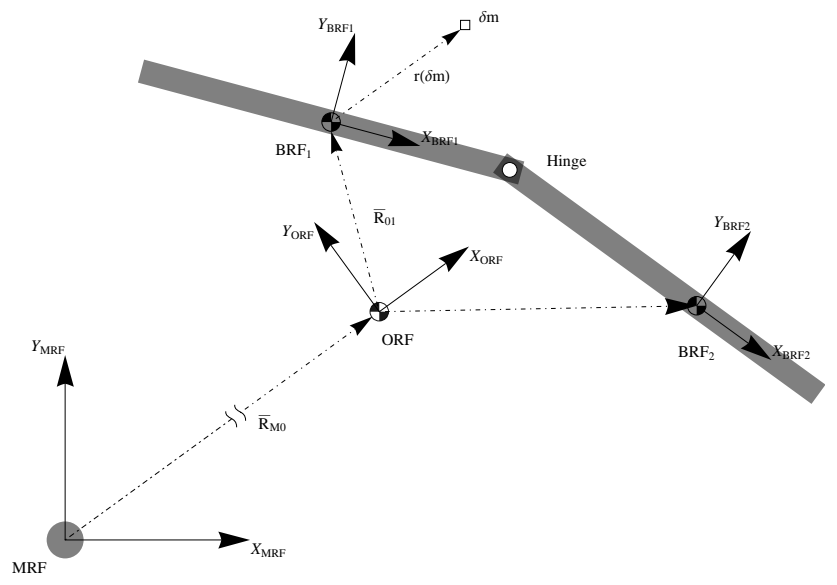

Figure 1: Illustration of the system and the reference frames used to describe it. 
In the past many methods have been developed to model multibody systems. In this paper we take inspiration from $,{ }^{7},{ }^{12},{ }^{20}$ and $^{21}$ to describe the problem and to derive the Hamiltonian function and the Hamiltonian form of the equations of motion. This is a convenient form to analyse the equilibria and their non-linear stability applying the Dirichlet's theorem.

The following reference frames will be used:

1. Main Reference Frame

2. Orbital Reference Frame

3. Body Reference Frame (one per body).

The Main Reference Frame $(M R F)$ is assumed to be inertial and fixed in space; its origin is the origin of the gravity field. The $\left.X\right|_{M R F}$ and $\left.Y\right|_{M R F}$ plane is assumed to be the orbital plane with the z-axis parallel to the orbital angular momentum vector.

The Orbital Frame $(O R F)$ is not inertial; its origin is the instantaneous overall center of mass of the multibody system. Its $\mathrm{x}$-axis is parallel to the position vector of the origin w.r.t. the $M R F$; the $\mathrm{z}$-axis is taken as parallel to the $\left.Z\right|_{M R F}$ and consequently the y-axis will be parallel to the local horizon such that it forms a right handed frame.

Every single body will be given its own reference frame $\left(B R F_{i}\right.$ - where the subscript $i$ is a number identifying the body), with the origin its center of mass and orientation set according to the principal axes of inertia.

\section{II.A. KINETIC ENERGY OF THE SYSTEM}

The particle mass of the $i$-th body is described by the following position vector:

$$
\begin{array}{r}
\vec{R}(\delta m)=\overrightarrow{R_{M 0}}+\mathbf{T}_{M 0} \overrightarrow{R_{0 i}}+\mathbf{T}_{M i} \overrightarrow{r_{i}}(\delta m)= \\
\overrightarrow{R_{M 0}}+\mathbf{T}_{M 0}\left(\overrightarrow{R_{0 i}}+\mathbf{T}_{0 i} \overrightarrow{r_{i}}(\delta m)\right)
\end{array}
$$

The following notation has been adopted:

- $\overrightarrow{R_{M 0}}$ position vector of the overall center of mass w.r.t. the $M R F$.

- $\overrightarrow{R_{0 i}}$ is the position vector of the i-th body center of mass w.r.t. the $O R F$

- $\vec{r}_{i}(\delta m)$ is the position vector of the infinitesimal mass $\delta m$ w.r.t. the $B R F$ of the rigid body it belongs to.

- $\mathbf{T}_{A B}$ is a coordinate transformation matrix which rotates a generic vector from reference frame $\mathrm{B}$ to the reference frame A. In particular the subscripts:

- M refers to the $M R F$

- 0 refers to the $O R F$

- i refers to the i-th $B R F$

Note that $\mathbf{T}_{M 0}$ depends on the orbital true anomaly $\nu$ only and $\mathbf{T}_{0 i}$ depends on the attitude angle of the i-th body $\theta_{i}$ only, as the problem is set as 2-D.

Moreover: $\mathbf{T}_{M i}=\mathbf{T}_{M 0} \mathbf{T}_{0 i}$.

Differentiating with respect to time and applying the inner product yields the kinetic energy of the $i$-th body as:

$$
\mathcal{K}_{i}=\frac{1}{2} \int_{\mathcal{B} i}<\dot{\vec{R}}(\delta m), \dot{\vec{R}}(\delta m)>\delta m
$$

Note that the rotation matrices satisfy the differential equation:

$$
\frac{d \mathbf{T}_{A B}(\psi)}{d t}=\mathbf{T}_{A B} \Omega_{A B}
$$


with $\Omega_{A B}$ a skew-symmetric matrix function of $\frac{d \psi}{d t}=\omega_{A B}$ only. Hence, $\omega_{0 i}$ is the angular spinning rate of the i-th body with respect to the $O R F$, while $\omega_{M 0}$ is the orbital rate. After some algebra and some simplifications due to the choice to place the origins of the reference frames over the center of masses of the system, $\mathrm{Eq}(2)$ yields:

$$
\begin{array}{rc}
2 \mathcal{K}= & m\left\|\dot{\vec{R}}_{M 0}\right\|^{2}+\hat{I}_{1}\left(\omega_{M 0}+\omega_{01}\right)^{2}+\hat{I}_{2}\left(\omega_{M 0}+\omega_{02}\right)^{2}+ \\
& -\quad 2 \epsilon<\vec{d}_{1 H}, T_{\theta} \vec{d}_{2 H}>\left[\omega_{01} \omega_{02}+\omega_{M 0}^{2}+\omega_{M 0}\left(\omega_{01}+\omega_{02}\right)\right]
\end{array}
$$

Where :

- $m\left\|\overrightarrow{\vec{R}}_{M 0}\right\|^{2}$ is the term related with the translational kinetic energy of the overall C.G.

- $m$ is the total mass of the multibody system

- $\epsilon=\frac{m_{1} m_{2}}{m}$

- $\vec{d}_{i H}=\vec{r}_{i}($ Hinge $)$ is the vector identifying the hinge position in the i-th $B R F$

- $I_{i}$ is the momentum of inertia of the i-th body w.r.t. its center of mass

- $\hat{I}_{i}=I_{i}+\epsilon\left\|\vec{d}_{i H}\right\|^{2} \quad$ for $\mathrm{i}=1,2$

- $\mathbf{T}_{\theta}=T_{01}^{-1} T_{02}$ which is dependent on the angle $\theta=\theta_{2}-\theta_{1}$

- $\hat{I}_{i}$ can be considered as the moment of inertia of each body relative to the hinge.

\section{II.B. THE GRAVITATIONAL POTENTIAL}

The effect of gravity on every single body is considered. Moreover, as the net effect of the gravity depends on the shapes and the attitudes of the bodies, some approximations will be introduced in order to obtain the simplest form of the potential functions. The derivation of the approximated form of the potential is inspired by a well known procedure, for instance see ${ }^{10}$ or. $^{22}$

For a mass particle $\delta m$, the gravity potential is:

$$
\delta \mathcal{U}=-\frac{G M}{|\vec{R}(\delta m)|} \delta m=-\frac{\mu}{|\vec{R}(\delta m)|} \delta m
$$

Where:

- $\mathrm{G}$ is the universal gravitational constant

- $M$ is the ideal mass generating the field

- $\mu=G M$

- $\vec{R}(\delta m)$ is the position vector of the particle $\delta m$ in the $M R F$

This potential will be expanded with respect to a point in space, identified by the position vector $\vec{q}$ in the $M R F$, such that $\vec{R}(\delta m)=\vec{q}+\vec{x}(\delta m)$, introducing the hypothesis

$$
\|\vec{q}\|=q \gg\|\vec{x}(\delta m)\|
$$

To simplify the writing of the series expansion we write $\vec{x}=\vec{x}(\delta m)$. This yields:

$$
\begin{gathered}
\frac{1}{|\vec{R}(\delta m)|} \cong \\
\frac{1}{q}-\frac{1}{q^{2}}<\vec{\gamma}, \vec{x}>+\frac{1}{q^{3}}\left(\frac{3}{2}<\vec{\gamma}, \vec{x}>^{2}-\frac{1}{2}<\vec{x}, \vec{x}>\right)+o\left[\frac{1}{q^{4}}\right]
\end{gathered}
$$

Two further hypotheses are considered:

i. the dimensions of the bodies allow their representation as one dimensional shapes;

ii. the density is constant $\rho(\vec{x})=\bar{\rho}$. 
It follows that: $\vec{r}_{i}(\delta m)=\{s, 0\}$ with $s$ a generic coordinate along the body. Moreover, following the convention used by Marsden et al., ${ }^{7}$ the position vectors of the hinge in their respective body frames will be taken as ${ }^{\text {a }}$

$$
\vec{d}_{H 1}=\left\{d_{1}, 0\right\} \quad ; \quad \vec{d}_{H 2}=\left\{-d_{2}, 0\right\}
$$

with $d_{1}, d_{2} \in \mathbb{R}^{+}$.

Manipulating Eq. (5) by expanding the inner products, integrating and applying the simplifications (i.) and (ii.), the two body problem potential function is:

$$
\mathcal{U}=\begin{gathered}
\mathcal{U}_{1}+\mathcal{U}_{2}= \\
-\mu\left\{\frac{m}{q}+\frac{3}{2} \frac{1}{q^{3}}\left[+2 \epsilon d_{2} d_{1} \cos \left(\theta_{1}\right) \cos \left(\theta_{2}\right)+\right.\right. \\
\left.+\cos \left(\theta_{1}\right)^{2} \hat{I}_{1}+\cos \left(\theta_{2}\right)^{2} \hat{I}_{2}\right]+ \\
\left.-\frac{1}{2}\left[\frac{\hat{I}_{1}+\hat{I}_{2}+2 \epsilon d_{1} d_{2} \cos (\theta)}{q^{3}}\right]\right\}
\end{gathered}
$$

\section{II.C. THE LAGRANGIAN FUNCTION}

Let the position vector of the ORF be expressed in cylindrical coordinates, $\vec{R}_{M 0}=\left\{R_{0} \cos (\nu), R_{0} \sin (\nu)\right\}$, so that

$$
q=\left\|\vec{R}_{M 0}\right\|=R_{0} \quad \text { and } \quad\left\|\dot{\vec{R}}_{M 0}\right\|^{2}=\dot{R}_{0}^{2}+\dot{\nu}^{2} R_{0}^{2}
$$

Also, let the name of the variables be changed to the following, in order to coincide with the general Lagrangian notation :

$\left\{R_{0}, \nu, \theta_{1}, \theta_{2}\right\}=\left\{q_{1}, q_{2}, q_{3}, q_{4}\right\}=\vec{q}$ and $\left\{\dot{R}_{0}, \omega_{M 0}, \omega_{01}, \omega_{02}\right\}=\left\{\dot{q}_{1}, \dot{q}_{2}, \dot{q}_{3}, \dot{q}_{4}\right\}=\overrightarrow{\dot{q}}$

The Lagrangian function of the system is:

$$
\begin{aligned}
& \mathcal{L}=\quad \mathcal{K}-\mathcal{U}= \\
& \frac{1}{2}\left\{m\left(\dot{q}_{1}^{2}+\dot{q}_{2}^{2} q_{1}^{2}\right)+\hat{I}_{1}\left(\dot{q}_{2}+\dot{q}_{3}\right)^{2}+\hat{I}_{2}\left(\dot{q}_{2}+\dot{q}_{4}\right)^{2}+\right. \\
& \left.+\alpha \cos \left(q_{4}-q_{3}\right)\left[\dot{q}_{3} \dot{q}_{4}+\dot{q}_{2}^{2}+\dot{q}_{2}\left(\dot{q}_{3}+\dot{q}_{4}\right)\right]\right\}+ \\
& +\mu\left\{\frac{m}{q_{1}}-\frac{1}{2} \frac{1}{q_{1}^{3}}\left[\hat{I}_{1}+\hat{I}_{2}+\alpha \cos \left(q_{4}-q_{3}\right)\right]+\right. \\
& \left.+\frac{3}{2} \frac{1}{q_{1}^{3}}\left[\alpha \cos \left(q_{3}\right) \cos \left(q_{4}\right)+\cos \left(q_{3}\right)^{2} \hat{I}_{1}+\cos \left(q_{4}\right)^{2} \hat{I}_{2}\right]\right\}
\end{aligned}
$$

Where $\alpha=2 \epsilon d_{1} d_{2}$.

Note that the variable $q_{2}$ does not appear explicitly in the Lagrangian and it is a cyclic variable. Its momentum is a conserved quantity of the system.

The Legendre transform is applied to the Lagrangian function to obtain the Hamiltonian function, where the conjugate momenta, $\vec{p}=\left\{p_{1}, p_{2}, p_{3}, p_{4}\right\}$, from Eq. (8) are defined as:

$$
\left\{\begin{array}{cccc}
p_{1}= & & m \dot{q}_{1} \\
p_{2}= & m q_{1}^{2} \dot{q}_{2}+\hat{I}_{1}\left(\dot{q}_{2}+\dot{q}_{3}\right)+\hat{I}_{2}\left(\dot{q}_{2}+\dot{q}_{4}\right) \\
& & +\frac{\alpha}{2}\left(2 \dot{q}_{2}+\dot{q}_{3}+\dot{q}_{4}\right) \cos \left(q_{4}-q_{3}\right) \\
p_{3}= & \hat{I}_{1}\left(\dot{q}_{2}+\dot{q}_{3}\right)+\frac{\alpha}{2}\left(\dot{q}_{2}+\dot{q}_{4}\right) \cos \left(q_{4}-q_{3}\right) \\
p_{4}= & \hat{I}_{2}\left(\dot{q}_{2}+\dot{q}_{4}\right)+\frac{\alpha}{2}\left(\dot{q}_{2}+\dot{q}_{3}\right) \cos \left(q_{4}-q_{3}\right)
\end{array}\right.
$$

The following notation can be adopted for convenience:

$$
\vec{p}=\mathbf{J} \overrightarrow{\dot{q}}
$$

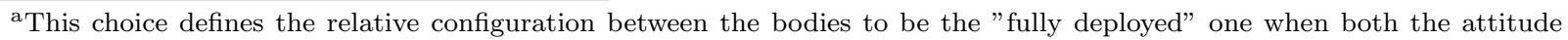
angles are zero. 
the Hamiltonian can then be written as:

$$
\begin{aligned}
\mathcal{H} & =\vec{p}^{T r} \mathbf{J}^{-1} \vec{p}-\mathcal{K}\left(\vec{q}, \mathbf{J}^{-1} \vec{p}\right)+\mathcal{U}(\vec{q})= \\
& =\frac{1}{2} \vec{p}^{T r} \mathbf{J}^{-1} \vec{p}+\mathcal{U}(\vec{q})
\end{aligned}
$$

\section{II.D. COMPLETE DYNAMICS OF THE SYSTEM}

The Hamiltonian equations of motion follow with: $\frac{\partial \mathcal{H}}{\partial \vec{p}}=\overrightarrow{\mathbf{I}}^{T r} \mathbf{J}^{-1} \vec{p} \quad$ where $\quad \overrightarrow{\mathbf{I}}^{T r}=\{1,1,1,1\}$ and : $\frac{\partial \mathcal{H}}{\partial \vec{q}}=\vec{p}^{T r} \frac{\partial \mathbf{J}^{-1}}{\partial \vec{q}} \vec{p}+\frac{\partial \mathcal{U}(\vec{q})}{\partial \vec{q}}$

yield:

$$
\begin{aligned}
& \left\{\begin{array}{lc}
\dot{q}_{1}= & \frac{p_{1}}{m} \\
\dot{q}_{2}= & \frac{p_{2}-p_{3}-p_{4}}{m q_{1}^{2}} \\
\dot{q}_{3}= & \frac{1}{m q_{1}^{2}}\left[-p_{2}+p_{3}\left(1+\frac{\hat{\hat{t}}_{2} m q_{1}^{2}}{\Delta}\right)+p_{4}\left(1-\frac{m q_{1}^{2} d_{1} d_{2} \epsilon \cos (\theta)}{\Delta}\right)\right. \\
\dot{q}_{4}= & \frac{1}{m q_{1}^{2}}\left[-p_{2}+p_{3}\left(1-\frac{m q_{1}^{2} d_{1} d_{2} \epsilon \cos (\theta)}{\Delta}\right)+p_{4}\left(1+\frac{\hat{I}_{1} m q_{1}^{2}}{\Delta}\right)\right.
\end{array}\right]
\end{aligned}
$$

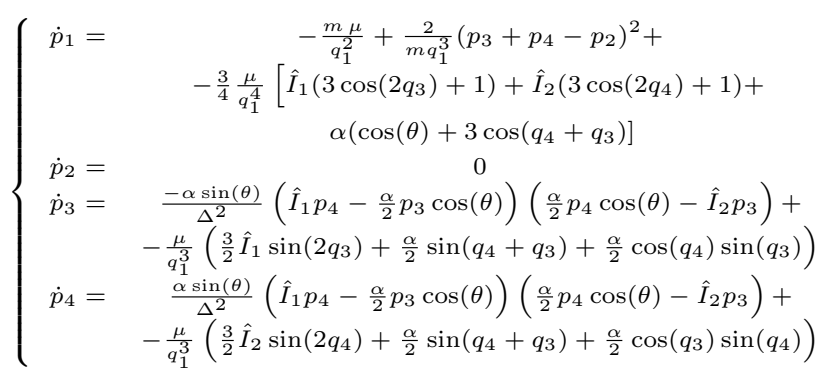

where $\Delta=\hat{\mathbf{I}}_{1} \hat{\mathbf{I}}_{2}-\left(\frac{\alpha}{2}\right)^{2} \cos (\theta)^{2}$. Some additional observations:

- The overall angular momentum is a conserved quantity of the problem (as expected) $)^{\mathrm{b}}$.

- With respect to the free case, the sum of the momenta $p_{3}+p_{4}$ is no longer constant. Indeed, there is a continuous exchange of momentum between the orbital and the attitude dynamics.

- Carrying out all the necessary calculations, it can be verified that there is a continuous exchange of energy between the orbital and the attitude dynamics.

The previous system of equations shows that the orbital dynamics and the attitude dynamics are coupled. However, an analysis of the magnitude order of the terms in the equations highlights a very small dependency of the orbital dynamics on the attitude, as long as the hypothesis on the length scale of the 2-body system holds (see Eq. (4)).

Specifically, this is motivated by the following considerations which hold in most of the practical cases, in particular for systems orbiting around the Earth :

$$
\begin{array}{rlrl}
o\left\{q_{1}\right\} \simeq o\{\mathcal{R}\}, o\{\mu\} & \simeq o\left\{\mathcal{R}^{2}\right\} ; & o\left\{\dot{q}_{2}\right\} \simeq o\left\{\sqrt{\frac{\mu}{\mathcal{R}^{3}}}\right\} \simeq o\left\{\mathcal{R}^{-1 / 2}\right\} \\
o\left\{p_{2}\right\} \simeq o\left\{\mathcal{R}^{2} \dot{q}_{2}\right\} \simeq o\left\{\mathcal{R}^{3 / 2}\right\} ; & o\left\{p_{3}\right\} \simeq o\left\{p_{4}\right\} \simeq o\left\{\left(m \ell^{2}\right) \omega_{\text {Spin }}\right\}
\end{array}
$$

so that

$$
\begin{aligned}
o\left\{p_{2}\right\} & \gg o\left\{p_{3}+p_{4}\right\} ; \\
\dot{p}_{1} \simeq \frac{m \mu}{q_{1}^{2}}+\frac{2}{m q_{1}^{3}}\left(p_{3}+p_{4}-p_{2}\right)^{2} ; & \dot{q}_{2} \simeq \frac{p_{2}}{m q_{1}^{2}}
\end{aligned}
$$

with

- $\mathcal{R}$ magnitude order of the semi-major axis of the orbit

- $m$ magnitude order of the mass of the system

\footnotetext{
${ }^{b}$ This is a very important element which also provides confirmation of the validity of the dynamic equations derived.
} 
- $\ell$ magnitude order of the length of the bodies

- $\omega_{\text {Spin }}$ magnitude order of the angular velocities of the bodies

The assumption that the orbital dynamics does not depend on the attitude dynamics allows us to focus on the attitude dynamics only.

\section{II.E. EQUILIBRIA}

Equilibria of the attitude motions are of the form:

$$
\begin{cases}q_{3}=\bar{q}_{3}=\text { const. } \quad \dot{q}_{3}=0=\text { const } . \\ q_{4}=\bar{q}_{4}=\text { const. } \quad \dot{q}_{4}=0=\text { const } .\end{cases}
$$

The condition on the dynamical system in order to satisfy these equations is:

$$
\begin{cases}\dot{q}_{3}=0 & \dot{p}_{3}=0 \\ \dot{q}_{4}=0 & \dot{p}_{4}=0\end{cases}
$$

In the system (11) the following set of solutions can be obtained:

$$
\left\{\begin{array}{l}
p_{3}=\frac{\bar{p}_{2}\left(\hat{I}_{1}+d_{1} d_{2} \epsilon \cos (\theta)\right)}{\hat{I}_{1}+\hat{I}_{2}+m q_{1}^{2}+2 d_{1} d_{2} \epsilon \cos (\theta)} ; \\
p_{4}=\frac{\bar{p}_{2}\left(\hat{I}_{2}+d_{1} d_{2} \epsilon \cos (\theta)\right)}{\hat{I}_{1}+\hat{I}_{2}+m q_{1}^{2}+2 d_{1} d_{2} \epsilon \cos (\theta)} ; \\
\theta=0+k \pi ; \\
\sin \left(q_{3}\right) \wedge \sin \left(q_{4}\right)=0 \vee \cos \left(q_{3}\right) \wedge \cos \left(q_{4}\right)=0
\end{array}\right.
$$

It is important to consider that in this set the value of the momenta depend on $q_{1}$. This directly implies that for circular orbits the condition will hold, however for elliptic orbits, where $q_{1}$ depends on time, there cannot be a natural stable equilibrium configuration. Note again that $q_{1}$ would never be exactly constant due to a continuous exchange of energy and momentum with the orbital dynamics. However, these particular effects are small enough to be considered negligible.

In particular, the system (15) defines eight equilibrium configurations:

\begin{tabular}{|c|c|c||c|c|c|}
\hline Config. Num. & $q_{3}$ & $q_{4}$ & Confi. Num. & $q_{3}$ & $q_{4}$ \\
\hline 1 & 0 & 0 & 5 & $\frac{\pi}{2}$ & $\frac{\pi}{2}$ \\
\hline 2 & $\pi$ & $\pi$ & 6 & $\frac{3 \pi}{2}$ & $\frac{\pi}{2}$ \\
\hline 3 & $\pi$ & 0 & 7 & $\frac{\pi}{2}$ & $\frac{3 \pi}{2}$ \\
\hline 4 & 0 & $\pi$ & 8 & $\frac{3 \pi}{2}$ & $\frac{3 \pi}{2}$ \\
\hline
\end{tabular}

Table 1: Classification of the Equilibria of the attitude dynamics

\section{II.F. STABILITY ANALYSIS}

Using the Dirichlet's Theorem ${ }^{\mathrm{c}}$, stable equilibria will be identified as critical points of the Hamiltonian. Thus its Hessian matrix is considered.

After the necessary calculations, the Hessian of the Hamiltonian at the equilibria can be expressed as a block diagonal matrix of the form:

$$
\left.H_{\mathcal{H}}\right|_{\text {Equilibria }}=\left[\begin{array}{cc}
\mathbf{D}_{1} & \mathbf{0} \\
\mathbf{0} & \mathbf{D}_{2}
\end{array}\right]
$$

The form of $\mathbf{D}_{2}$ is such that its eigenvalues will always be real and positive. $\mathbf{D}_{1}$ will have real and positive eigenvalues for two configurations only, thus making the Hessian positively definite. The non-linearly stable equilibria are:

${ }^{\mathrm{c}}$ Where the Hamiltonian function is considered as a Lyapunov function, e.g. $\operatorname{see}^{23}$ 
- $q_{3}=0 \wedge q_{4}=0$

- $q_{3}=\pi \wedge q_{4}=\pi$

\section{II.G. DYNAMICAL SYSTEM ANALYSIS}

In the previous sections of the paper a model has been derived and analysed using the Hamiltonian function. However, a global description of the system behaviour has not been provided yet. To this end, different representations of the dynamics will be shown using various analysis tools. The dynamic equations (11) are integrated using a standard integrator in Wolfram Mathematica. The Hamiltonian and the total angular momentum are introduced in the differential equations set as extra variables to be integrated. This has been preferred to the integration of a reduced system in order to keep control of the numerical errors and to drive the accuracy of the integration always below a maximum relative error of an order of $o\left(10^{-12}\right)$ on the initial values of the conserved quantities.

The following conditions will apply for the solutions presented in the next sections:

- The system is considered orbiting around the Earth;

- The nominal orbit is circular with an altitude of $300 \mathrm{Km}$ from the Earth's surface;

- The parameters defining the bodies are

\begin{tabular}{||c|c||}
\hline Length & Mass \\
\hline $2 \mathrm{~m}$ & $10 \mathrm{Kg}$ \\
\hline $2 \mathrm{~m}$ & $10 \mathrm{Kg}$ \\
\hline \hline
\end{tabular}

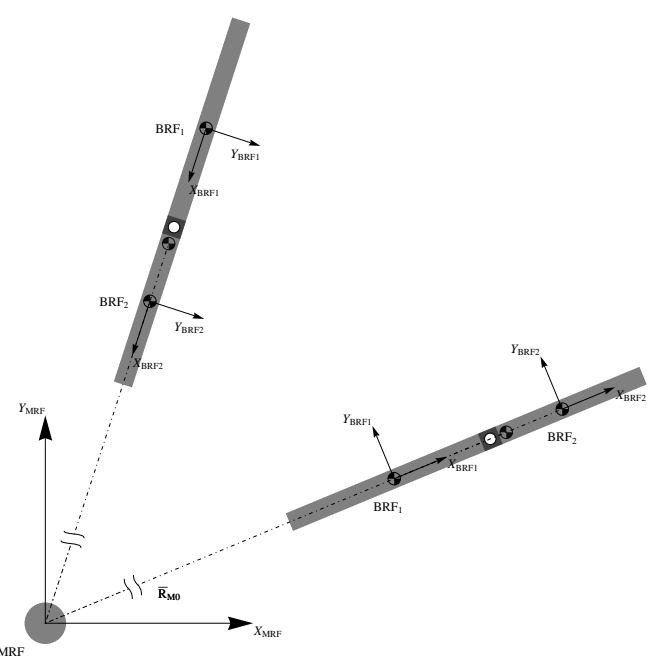

Figure 2: Illustration of the stable equilibrium configurations of the system. They apply only for circular orbits.

\section{II.G.1. PHASE PORTRAITS}

There are many perspectives dynamical systems can be observed from. One of most elementary, is the description of the natural evolution through phase plots, sections of the trajectories in the state space, starting from some particular initial condition. Although phase portraits offer a local and quite limited description, especially for high-dimensional systems, they are an invaluable tool in studying dynamical systems, revealing information such as whether an attractor, a repeller or a limit cycle is present.

An accurate observation of this planar two orbiting rigid body problem, highlights three different kinds of motion.

The following phase portraits provide a concise description of these types of motions. Figures 3a, 4a and $5 \mathrm{a}$ are the projections of the trajectories of the system in the $\left\{q_{3}, q_{4}\right\}$ plane. Figures $3 \mathrm{~b}, 4 \mathrm{~b}$ and $5 \mathrm{~b}$ are the projections of the trajectories of the system in the $\left\{q_{3}, \dot{q}_{3}\right\}$ plane.

Figures $3 \mathrm{a}$ and $3 \mathrm{~b}$ are generated by an initial condition "relatively close" to the stable equilibrium point. It is easy to identify a periodic motion.

Figures $4 \mathrm{a}$ and $4 \mathrm{~b}$ are generated by an initial condition "relatively far" from both the stable and the unstable equilibrium points. Some regularity is shown in this motion. Although It is definitively not possible 


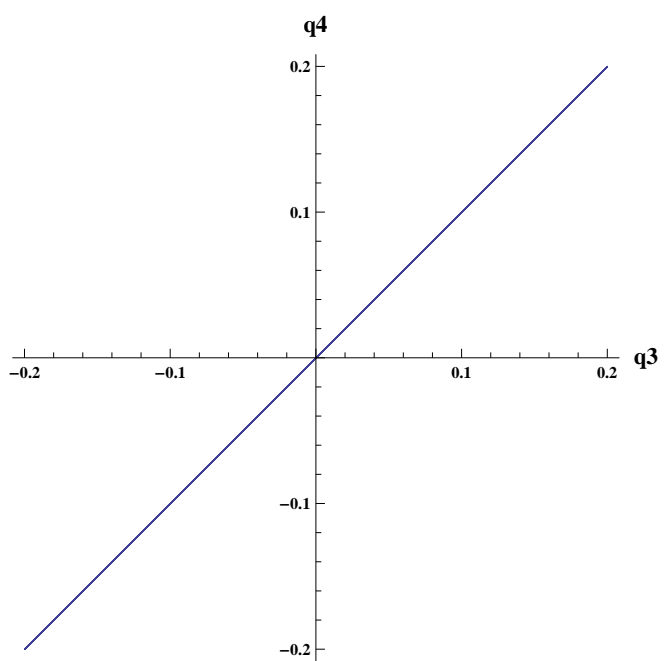

(a) Phase plot in the plane $\left\{q_{3}, q_{4}\right\}$. Case 1 : the behaviour exhibited is clearly regular and periodic. The motion repeats itself over a line moving from left to right then from right to left.

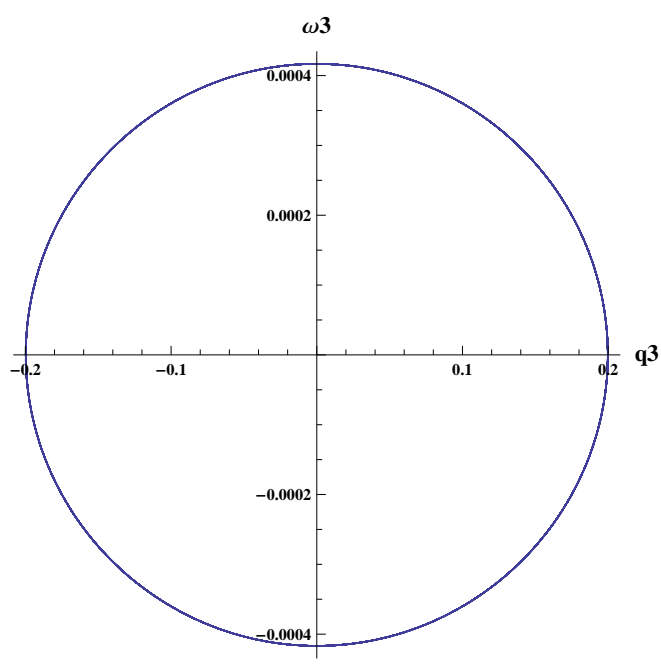

(b) Phase plot in the plane $\left\{q_{3}, \dot{q}_{3}\right\}$. Case 1 : the behaviour exhibited is clearly regular and periodic. The motion repeats itself over a circle from this perspective and it moves clockwise.

Figure 3: Case 1, phase plots

to define it as periodic, it may be identified as "quasi-periodic". By this we mean that the system regularly does not evolve on the same trajectory, but on a trajectory "close" to the previous one, thus showing irregular periodicity and remaining confined in a limited region of the phase space.

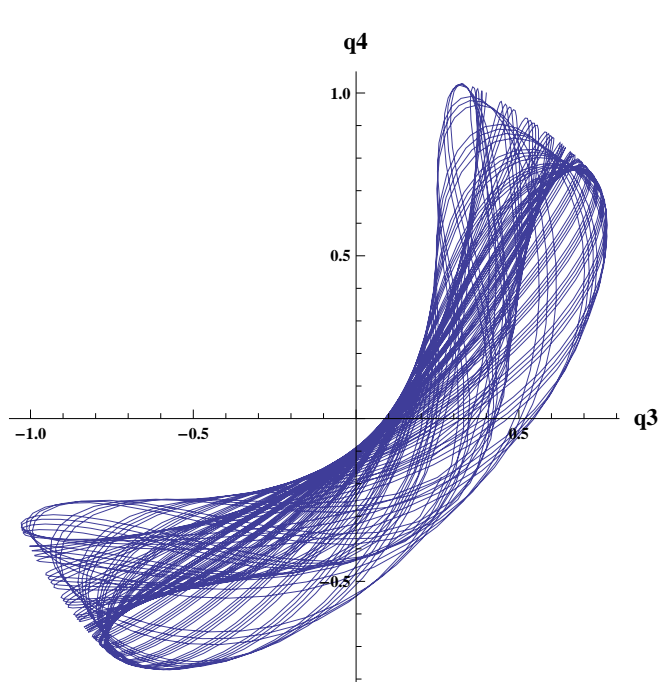

(a) Phase plot in the plane $\left\{q_{3}, q_{4}\right\}$. Case 2: the behaviour exhibited is not fully periodic but it is regular. Qualitatively it can be identified as quasi-periodic.

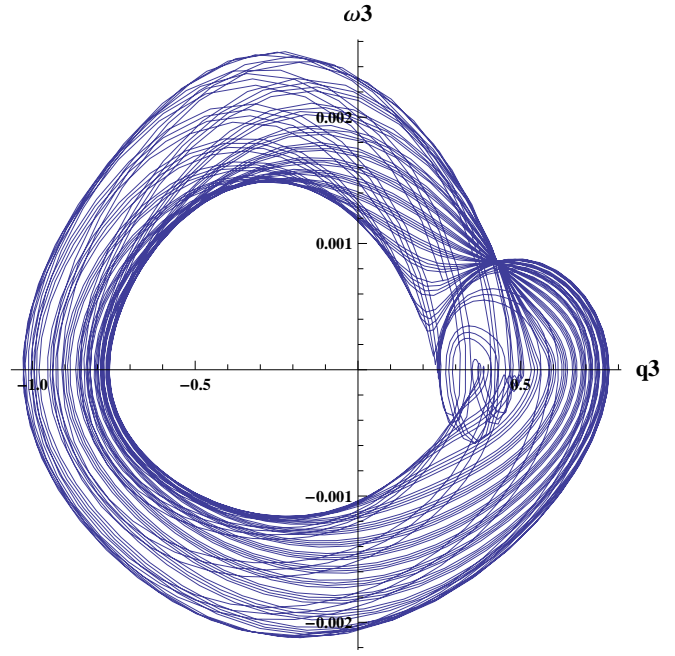

(b) Phase plot in the plane $\left\{q_{3}, \dot{q}_{3}\right\}$. Case 2: this plot shows a regular motion even though not periodic. Its appearance may suggest the presence of a strange attractor and, indeed, it could be affirmed that the behaviour is "weakly" chaotic. This motion is classified here as quasi-periodic.

Figure 4: Case 2, phase plots 
Figure $4 \mathrm{~b}$ is particularly interesting as it may suggest the presence of a chaotic attractor ${ }^{\mathrm{d}}$. However, observing motions generated by initial conditions in the neighborhood of this particular case (Case 2), high sensitivity on the initial conditions has not been shown and qualitatively similar trajectories have been observed $^{\mathrm{e}}$. This last elements agree with the identification of this behaviour as quasi-periodic.

Figures $5 \mathrm{a}$ and $5 \mathrm{~b}$ are generated starting with an initial condition "relatively close" to the unstable equilibrium point. The motion becomes highly irregular. Motions starting in this area evolve on completely different trajectories and do not remain confined. This clearly suggests a fully chaotic behaviour.

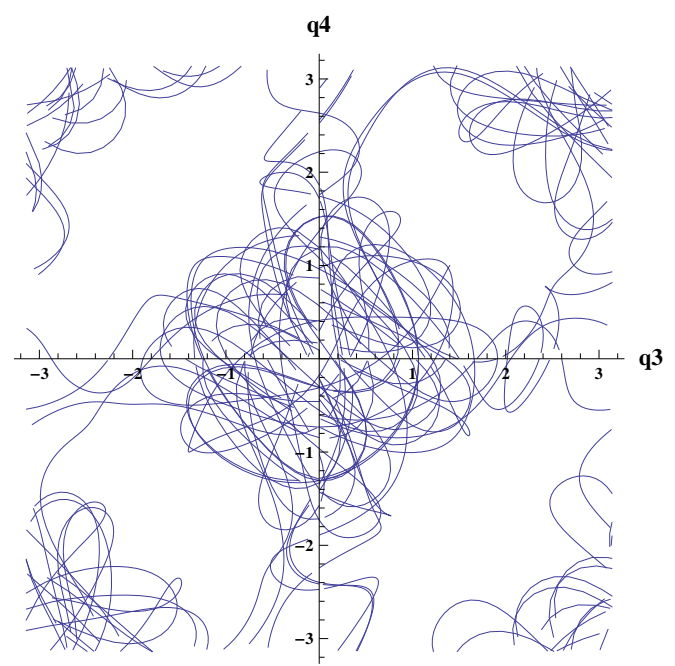

(a) Phase plot in the plane $\left\{q_{3}, q_{4}\right\}$. Case 3: the behaviour exhibited is clearly irregular and fully aperiodic. It is identified as chaotic.

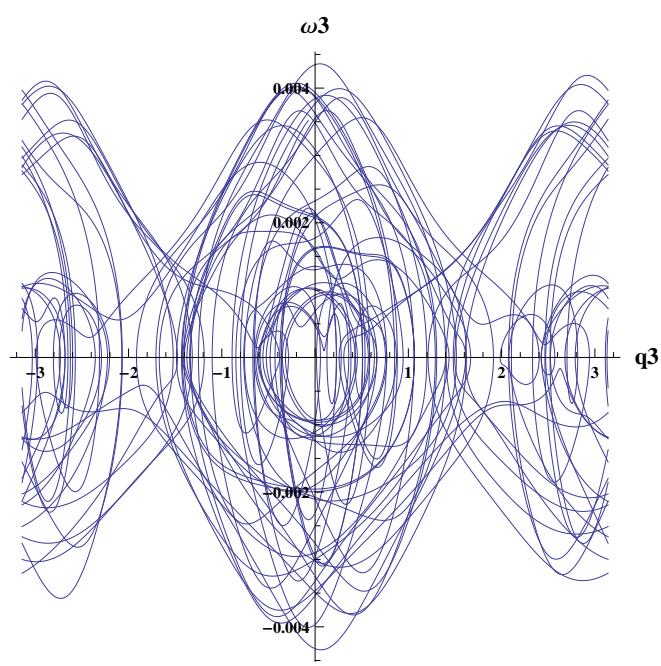

(b) Phase plot in the plane $\left\{q_{3}, \dot{q}_{3}\right\}$. Case 3: the behaviour exhibited is clearly irregular and fully aperiodic. It is identified as chaotic.

Figure 5: Case 3, phase plots

\section{II.G.2. POINCARÉ SECTIONS}

In order to further investigate the same system's evolutions from another perspective, Poincaré sections are used.

Poincaré sections are a standard technique to inspect complex systems. These are projections of phase spaces where a snap shot is taken whenever the trajectory intersects a prescribed plane in the phase space. Consequently, the Poincaré mapping ${ }^{\mathrm{f}}$ is a collection of points sampled in a regular way from the phase portraits. For further details on Poincaré maps, see ${ }^{24}$ or. $^{25}$

For this problem Poincaré sections are constructed sampling the state of the system when the condition $\left\{\theta_{1}=0, \omega_{1} \geq 0\right\}$ is verified.

Figure $6 \mathrm{a}$ is a Poincaré section, in the plane $\left\{q_{4}, \dot{q}_{4}\right\}$ of the regular motion shown in figures 3a and 3b. The whole trajectory is represented here simply by two dots. This provides further evidence of the fact that the system evolves naturally on a periodic trajectory. It should be noted that no dissipative forces have been included in the model.

Consider now Case 2. Figure 6b clearly shows regularity. The system's natural evolution is completely described by two closed curves supporting the identification of the motion as quasi-periodic.

Finally, in the Poincaré section related to Case 3, any sort of regularity disappears and the points cover an apparent random shaped area rather than being ordered along a curve.

This kind of behaviour can be recognised in the Poincaré sections of many systems as the double pendulum, the three body system or the Hénon-Heiles equations.

\footnotetext{
${ }^{\mathrm{d}}$ See for instance the typical phase plot of a Lorentz attractor.

ePresumably, this particular motion is characterized by irrational frequencies, typical element of quasi-periodicity.

${ }_{\mathrm{f}}^{\mathrm{A}}$ Also referred as a stroboscopic technique
} 


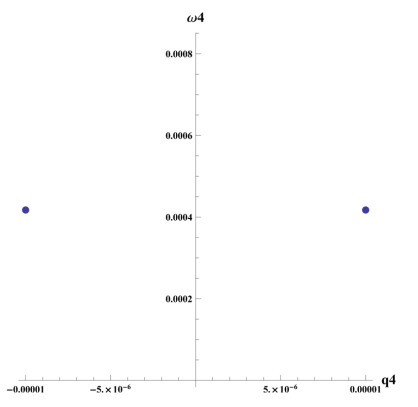

(a) Poincaré section in the plane $\left\{q_{4}, \dot{q}_{4}\right\}$. This plot refers to Case 1 and it constitutes a further evidence of the periodicity of the orbit as the whole motion is represented by two points only.

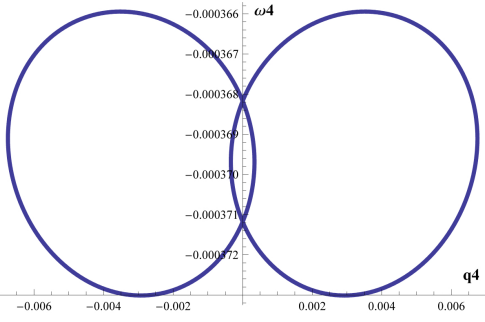

(b) Poincaré section in the plane $\left\{q_{4}, \dot{q}_{4}\right\}$. This plot refers to Case 2 and it constitutes a clear evidence of the quasi-periodicity of the orbit. The motion evolves on two closed curves.

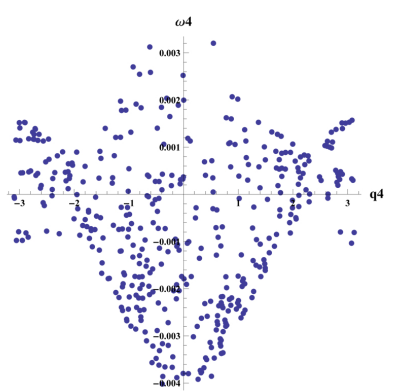

(c) Poincaré section in the plane $\left\{q_{4}, \dot{q}_{4}\right\}$. This plot refers to Case 3 and it constitutes a clear evidence of the fact that chaotic behaviour is exhibited. The motion is not represented by any regular curve but it is dense over an area.

Figure 6: Case 1, 2 and 3; Poincaré section

\section{II.G.3. LYAPUNOV CHARACTERISTIC EXPONENTS}

LCEs are a concept introduced by Lyapunov in the early 20th century but largely addressed by the academic community from the 1960 (refer to $,{ }^{26},{ }^{2728}$ and ${ }^{29}$ for an overview). Roughly speaking they are a measure of the mean exponential rate of divergence of system's trajectories. A single LCE is calculated from a specific initial condition, thus it provides a local description of the behaviour of the system. This piece of information is contained in a real number and therefore a density map of the LCEs can be illustrated, collecting a quantity of information in an image. Moreover, this provides qualitatively a description from a wider point of view. Depending on the total dimension of the system, a set of maps can be generated. This gives an almost-global description of the system's behaviour which can be interpreted at a glance. The calculation of a Lyapunov Exponent can rarely be done analytically, thus only with the introduction of modern computers the LCEs have become an effective tool in studying complex dynamical systems. This makes them a relatively new technique which, in particular, have found application in astrodynamics, ${ }^{18,30}$ and in fluid dynamics, ${ }^{15,16}$

At the moment many methods have been proposed to calculate the maximum LCE (or the whole spectrum). In this paper we use the Gram-Schmidt orthonormalisation method,. ${ }^{31}$ This is our preferred choice as it is complete, reliable, continuous and stable as well as easy to implement in the same code used to integrate the dynamics of the system.

Specifically, we use the following definition, referring to. ${ }^{31}$ Given the flow along an orbit $x(t)=\phi^{t}\left(x_{0}\right)$, the evolution of a nearby orbit could be expressed as $x^{\prime}(t)=\phi^{t}\left(x_{0}+\delta x_{0}\right)=x(t)+u(t)$. Given $x^{\prime}(0)=x_{0}+u_{0}, u(t)$ can be defined using the tangent map $u(t)=M_{x_{0}}(t) u_{0}$, with the transition matrix $M_{x_{0}}(t)=\partial \phi^{t}\left(x_{0}\right) / \partial x_{0}$. The spectrum of the matrix $M_{x_{0}}^{T}(t) M_{x_{0}}(t)$ is real and positive and the generic eigenvalue can be defined as $\mu_{k}^{2}(t)$. Then, the Lyapunov Exponent associated with the initial condition $x_{0}$ can be defined as:

$$
\lambda_{k}=\lim _{t \rightarrow+\infty} \frac{1}{t} \log \mu_{k}(t)
$$

LCEs are always a real number. Roughly speaking, when the LCE associated to one of the configuration variables is positive, two very close initial conditions will evolve exponentially diverging along that direction. Similarly, a negative exponent represents converging motions and a zero exponent can be considered representative of invariant motions.

Figure 7 shows the evolution in time of LCEs associated to the variable $q_{3}$ in Case 1 (continuous line at the bottom) and Case 3 (dashed line at the top). By definition, LCEs are exact only for infinite time of integration, however, note that the LCE decreases exponentially to its value. Moreover, probably due to numerical errors, the right tail of the LCE curve is not flat but presents periodic oscillations. This attitude is maintained in all the cases calculated. In order to overcome these problems and to provide a reliable LCE 
value, two actions have been taken: first

i. a minimum time integration of 200000 seconds is used;

ii. only the last 10000 seconds are considered and over this range a mean value is calculated.

Following this procedure, LCEs are used as a further tool to investigate the dynamics of the system. Attention here is given to the attitude dynamics only. Table 2a summaries the values obtained for Cases 1 , 2 and 3 :

Once normalised with respect to the magnitude of the largest, the LCEs can be compared to one another, see Tab. $2 \mathrm{~b}$.

Note that:

- the first case ${ }^{\mathrm{g}}$ shows one negative LCE and another one two orders of magnitude smaller than the ones of the $3 \mathrm{rd}$ case.

- the second case ${ }^{\mathrm{h}}$ shows LCEs one magnitude order smaller than the ones of the 3rd case

The presence of different orders of magnitudes together with the results of the phase portraits and the Poincaré sections prove that different kinds of motion are related to clearly distinct LCEs. This implies that different regions of motions will be shown in a density map: see fig. 8. Here the colour of each dot depends on the value of the LCE calculated from the initial conditions corresponding to the coordinates of the dot itself. Brighter colors are associated with higher LCEs. In this case initial angular velocities are always null and therefore nonlinearly stable equilibria appear explicitly in the map. As verified from the Eq. (15), equilibria are located at the center of the darkest regions surrounded by a white ring. These white rings practically define the regular regions around the stable equilibrium points ${ }^{\mathrm{i}}$.

\begin{tabular}{||c|c|c||}
\hline Case & $\lambda 3$ & $\lambda 4$ \\
\hline 1st & 0.55 & -0.18 \\
\hline 2nd & 1.93 & 1.68 \\
\hline 3rd & 6.88 & 1.60 \\
\hline \hline
\end{tabular}

\begin{tabular}{||c|c|c||}
\hline Case & $\lambda 3$ & $\lambda 4$ \\
\hline 1st & 0.08 & -0.02 \\
\hline 2nd & 0.28 & 0.22 \\
\hline 3rd & 1 & 0.23 \\
\hline
\end{tabular}

(a) LCEs values. Values must be scaled of a factor $10^{-5}$

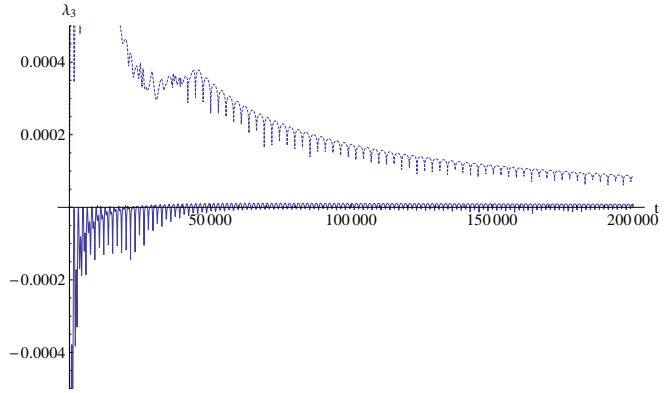

Figure 7: Evolution in time of the Lyapunov Characteristic Exponent $\lambda_{3}$ associated with the configuration variable $q_{3}$ during the system integration. It is observed that the trend exhibited is qualitatively exponential. Thus, a minimum time of integration is required in order to let the LCE settle toward its value. The curve at the bottom refers to Case 1 , the one at the top refers to Case 3.

Table 2: Case 1, 2 and 3; LCE

Shadowed areas surrounding the rings, in this particular problem, are associated with quasi-periodic regions. Far from those shadowed regions, the colour of the map becomes much brighter and chaotic regions arise. Both these statements are supported by the results of direct inspections. In particular, Case 1, 2 and 3 belong, respectively, to the regular, the quasi-regular and the irregular region of motion. In fig. 8, the initial conditions used to generate these specific cases are marked by the numbers 1,2 and 3 .

\footnotetext{
gThe periodic motion.

hAssumed to be "quasi-periodic".

${ }^{\mathrm{i}}$ Analogous to separatrices in phase plots.
} 
The richness of information enclosed in the map has a significant value as it provides, in a single image, a quasi-global description of the system's behaviour. As each map is related to only two configuration variables, in order to obtain a global description of the system a collection of maps is required so to cover the all phase space. Moreover, all this comes at the cost of a relatively moderate numerical computational effort and hence the use of this technique of investigation appears to be particularly promising and useful in the field of multibody space systems.

These results are of extreme advantage of the system design, similarly to fluid-dynamics and astrodynamics. Indeed, it can be used to drive the overall system design, for instance, in such a way that the equilibria are placed in the most convenient configurations. Indeed, it is well known that the whole motion of free-flying robotic systems depends on the position of the overall center of mass, which, in turn, is heavily influenced by the spatial disposition of the main elements of the system. Often, such design follows schemes defined a-priori but this method of analysis can be used to advance new solutions, which will ultimately enhance the efficiency of the system and its performances.

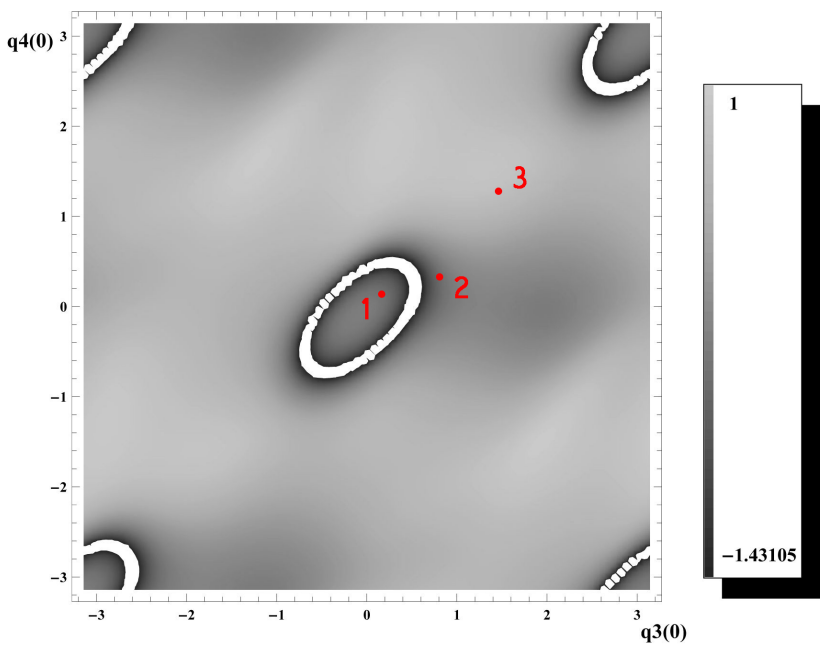

Figure 8: LCE density map of the planar gravitational two-body problem. Every dot of the plot represents an initial condition $\left\{q_{3}(0), q_{4}(0), 0,0\right\}$. Its color is associated with the value of the LCE calculated. Darker colors represent lower LCE values. Because the initial angular velocities are both null, this map can be taken as an equilibria stability analysis as well. The markers 1,2 and 3 correspond to the initial conditions used to generate the three cases showed in the first part of the paper.

Furthermore, LCEs maps can be used to gain insight into optimal control strategies which could be tailored to specific applications in order to gain the best performances from the systems. For example, LCE maps could be generated to describe the motion of the end-effector in order to identify regular-motion regions where sections of an invariant motion can be tracked with a simple controller.. Viceversa, the identification of a chaotic region as an operative point of work can motivate the use of a chaotic controller.

In this work a simple model was chosen and the utility of LCE maps has been demonstrated without any significant mathematical manipulation. However, this is not guaranteed for more complex cases and will be the subject of the future research.

\section{CONCLUSIONS}

The use of multibody systems in space engineering, e.g. automatic robotic systems or space manipulators, sets problems of smart system design, efficient maneuvering and nonlinear control strategies as key challenges to face. To this end, a study of the nonlinear underlying dynamics is fundamental and it paves the way to new design solutions. However, because of the dynamic complexity, a global analysis is impossible in most of the practical cases. To tackle this, the use of different tools potentially allows to gain a representation as complete as possible.

This paper uses Lyapunov Characteristic Exponents maps applied to a space multibody system to analyse different qualitative behaviours, reducing the order of the problem and condensing quantities of information into lower-dimensional images.

This tool, new to the field of space multibody systems, potentially has a significant value, particularly when supported by other methods of inspection. LCE maps allow quick visualisation of the nonlinearly stable equilibria as well as chaotic regions, regular or quasi-regular regions of motion. This richness of information, which comes at the cost of a relatively moderate numerical computational effort, is of considerable advantage to system design. 


\section{References}

${ }^{1}$ M.A. Diftler, R. A., "Robonaut: A Robotic Astronaut Assistant," Proceeding of the 6th International Symposium on Artificial Intelligence and Robotics $\&$ Automation in Space: i-SAIRAS, Canadian Space Agency, St-Hubert, Quebec, Canada, 2001.

${ }^{2}$ E. Coleshilla, L. Oshinowoa, R. R. B. B. D. R. S. S., "Dextre: Improving maintenance operations on the International Space Station," Acta Astronautica, Vol. 64, 2009.

${ }^{3}$ S. Ali A. Moosavian, E. P., "Free-Flying robots in space: an overview of dynamics modeling, planning and control," Robotica, 2007.

${ }^{4}$ Dubowsky, S., "A perspective of the advancements of robotic system during the past 15 years," Annual Reviews in Control, 1998.

${ }^{5}$ L. Pedersen, D. Kortenkamp, D. W. I. N., "A Survey of Space Robotics," Proceeding of the 7th International Symposium on Artificial Intelligence, Robotics and Automation in Space (i-SAIRAS-03), 2003.

${ }^{6}$ P.J. From, K.Y. Pettersen, J. G., "Singularity-free dynamic equations of spacecraft-manipulator systems," Acta Astronautica, 2011.

${ }^{7}$ Sreenath, N., O. Y. K. P. M. J., "The Dynamics of Coupled Planar Rigid Bodies. Part I Reduction, Equilibria and Stability," Dynamics and Stability of Systems, 1998.

${ }^{8}$ Sreenath, N., O. Y. K. P. M. J., "The Dynamics of Coupled Planar Rigid Bodies. Part II Bifurcations, Periodic Solutions, and Chaos," Journal of Dynamics and Differential Equations, 1989.

${ }^{9}$ Grossman, R., K. P. M. J., "Dynamics of two coupled three dimensional rigid bodies," Tech. rep., University of Maryland, 1987. 1993.

${ }^{10}$ Bogoyavlenskil, O. I., "Integrable Problems of the Dynamics of Coupled Rigid Bodies," Russian Acad. Sci. Izv. Math.,

${ }^{11}$ Guerman, A., "Equilibria of Multibody Chain in Orbit PLane," Journal of Guidance, Control and Dynamics, 2003.

${ }^{12}$ A.K. Sanyal, A. Bloch, N. H. M., "Dynamics of multibody systems in planar motion in a central gravitational field," Journal of Dynamicsal Sytems, 2004.

${ }^{13}$ Sanyal, A., Dynamics and control of multibody systems in central gravity, Ph.D. thesis, Department of Aerospace Engineering, University of Michigan, Ann Arbor, 2004.

${ }^{14}$ G.W.Patrick, Two axially symmetric coupled rigid bodies: relative equilibria, stability, bifurcations, and a momentum preserving symplectic integrator, Ph.D. thesis, Department of Mathematics, University of California, Berkeley, 1995.

${ }^{15}$ T. Peacock, G. H., "Lagrangian coherent structures, the hidden skeleton of fluyd flows," Physics Today, 2013.

${ }^{16}$ C. Senatore, S. R., "Detection and characterization of transport barriers in complex flows via ridge extraction of the finite time Lyapunov exponent field," International Journal for Numerical Methods in Engineering, 2011.

${ }^{17}$ C.R. Short, K.C. Howell, X. T., "Lagrangian Coherent Structures in the Restricted Three-body Problem," 21st AAS/AIAA Space Flight Mechanics Meeting, 2011.

${ }^{18}$ Gawlik, E.S., M. J. E. D. T. P. and Campagnola, S., "Lagrangian coherent structures in the planar elliptic restricted three-body problem," Celestial Mechanics and Dynamical Astronomy, 2009.

${ }^{19}$ M. Cencini, F. G., "Lyapunov analysis: from dynamical systems theory to applications," Journal of Physics A: Mathematical and Theoretical, 2013.

${ }^{20}$ Papadopoulos, E., On the Dynamics and Control of Space Manipulators, Ph.D. thesis, Department of Mechanical Engineering, Massachussetts Intitute of Technology, 1990.

${ }^{21}$ P. Santini, P. G., "General background and approach to multibody dynamics for space applications," Acta Astronautica, 2009.

${ }^{22}$ P. Santini, P. G., "Dynamics of multibody systems in space environment; Lagrangian vs. Eulerian approach," Acta Astronautica, 2003.

${ }^{23}$ B.Rink, T. T., "Stability in Hamiltonian Systems: Applications to the restricted three-body problem," Tech. rep., Mathematisch Instituut, Utrecht University, 2003.

${ }^{24}$ J.M.T. Thompson, H. S., Nonlinear Dynamics and Chaos, John Wiley and Sons, 1986.

${ }^{25}$ Hilborn, R. C., Chaos and nonlinear dynamics : an introduction for scientists and engineers, New York, Oxford University Press, 1994.

${ }^{26}$ G. Benettin, L. Galgani, A. G. J.-M. S., "Lyapunov Characteristic Exponents for Smooth Dynamical Systems and for Hamiltonian Systems; A method for Computing All of Them'. Part I: Theory." Meccanica, 1979.

${ }^{27}$ G. Benettin, L. Galgani, A. G. J.-M. S., "Lyapunov Characteristic Exponents for Smooth Dynamical Systems and for Hamiltonian Systems; A method for Computing All of Them'. Part II: Numerical Application." Meccanica, 1979.

${ }^{28}$ Wolf, A., Quantifying chaos with Lyapunov exponents, Chaos, Princeton University Press, 1986.

${ }^{29}$ G.L. Baker, J. G., Chaotic Dynamics: An Introduction, Cambridge University Press, 1990.

${ }^{30}$ Perez, D., G. G. M. J., "Detecting Invariant Manifolds Using Hyperbolic Lagrangian Coherent Structures," IAA Conference on Dynamics and Control of Space Systems, 2012.

${ }^{31}$ F. Christiansen, H. R., "Computing Lyapunov spectra with continuous Gram-Schmidt orthonormalization," Nonlinearity, 1997. 